\title{
Diagnosis and treatment of malaria
}

\author{
Despite accurate diagnostic tests over-diagnosis and presumptive treatment \\ are common practice
}

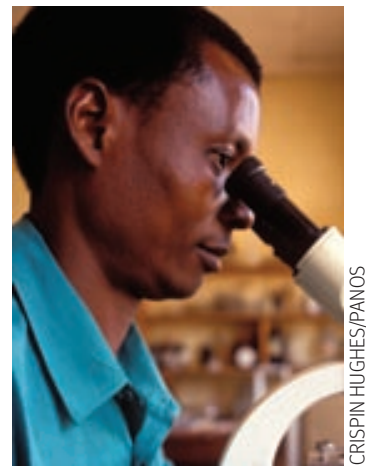

RESEARCH p 403

Ambrose 0 Talisuna assistant commissioner of health services, Uganda Ministry of Health, Epidemiological Surveillance Division, PO Box 7272, Kampala, Uganda

atalisuna@afsat.com

Denise Njama Meya research coordinator Makerere UniversityUniversity of California San Francisco Malaria Research Collaboration, PO Box 7475 ,

Kampala, Uganda

Competing interests: None declared.

Provenance and peer review: Commissioned; not externally peer reviewed.

BMJ 2007;334:375-6 doi: 10.1136/bmj.39126.485370.BE
Malaria is a major public health problem and is endemic in about 107 countries. The symptoms of uncomplicated malaria are non-specific and similar to many other disease syndromes, including minor viral illnesses. People living in areas where malaria is endemic are often familiar with these symptoms and frequently diagnose themselves, so that over-diagnosis is widespread.

Prompt and accurate diagnosis of malaria is important for effective case management and if implemented well should reduce mortality from this disease. ${ }^{2}$ High sensitivity of diagnosis is crucial, and high specificity could reduce unnecessary treatment and improve the diagnosis of other febrile illness. ${ }^{3}$ In this week's $B M J$, a randomised controlled trial by Reyburn et al assesses the effect of rapid diagnostic tests compared with microscopy for guiding treatment of acute febrile illness in outpatients in Tanzania. ${ }^{4}$

Light microscopy and rapid diagnostic tests are the two most commonly used methods of confirming a diagnosis of malaria. Microscopy, the gold standard, has several advantages including low cost and high sensitivity and specificity when used by well trained staff. Rapid diagnostic tests (which detect parasite antigens) are easier to perform by staff with basic training, have less waiting time and indirect costs, but are relatively more expensive. A recent decrease in their cost may make it possible to increase their use in subSaharan Africa.

Despite the availability of these two methods, presumptive treatment of malaria (without laboratory confirmation) remains common practice. ${ }^{5}$ Reyburn and colleagues found massive over-diagnosis of malaria. ${ }^{4}$ Surprisingly, rapid diagnostic tests combined with basic training did not reduce over-treatment for malaria. Of the 1193 and 1204 patients with complete data who were randomised to rapid diagnostic tests and microscopy, respectively, only 52\% and 50\% had a correct prescription. More than half the prescriptions for antimalarial drugs were given to people who had negative test results (blood smear or rapid diagnostic test). ${ }^{4}$ Furthermore, children with negative results of rapid diagnostic tests were more likely to be treated for malaria than those with a negative smear.

In the era of artemisinin combination treatments, we urgently need to improve parasitological confirmation of the diagnosis of malaria. Reasons include the relatively high cost of such treatments, which makes unnecessary treatment unsustainable; the need for better care in people who have parasite positive tests; the need to reduce the risk of adverse events and drug use to limit the selection of drug resistant parasites; the need for more robust health information; and the need to identify people with parasite negative tests in whom another diagnosis must be sought.

Reyburn and colleagues found that health workers continue to treat people for malaria even if the diagnostic test is negative,${ }^{4}$ emphasising the need to change the behaviour of such workers. However, they also found that this is difficult to do. The practice of treating patients with a negative test may be due to traditional teaching in medical schools, which promotes treatment on the basis of the health worker's index of suspicion, and also due to ambiguous phrases in some national guidelines.

The World Health Organization's generic treatment guidelines recommend parasitological confirmation of the diagnosis of malaria where malaria transmission is low, moderate, or unstable. ${ }^{6}$ In settings where the incidence of malaria is low, WHO recommends that health workers should be trained to identify patients who have been exposed to malaria before they carry out a parasitological test. In stable high transmission settings, where malaria is a common cause of febrile illness in children, WHO recommends that antimalarial drugs should be given to children with fever $\left(\geq 37.5^{\circ} \mathrm{C}\right)$ or a history of fever that has no other obvious cause. In children 5 years old and above, in pregnant women, and in settings with a high prevalence of HIV a diagnosis should have parasitological confirmation.

The WHO guidelines do not state that a patient with a negative test should be treated for malaria. However, some countries, such as Uganda, have adopted phrases like, "Any patient with fever or a history of fever within 24 hours without evidence of other disease should be treated for malaria even with a negative blood smear for malaria parasites." "Such recommendations are aimed at increasing antimalarial coverage and potentially reducing the risk of progression to severe disease and death.

However, recent evidence from the field does not support such practices. In Kenya, even with imperfect conditions for microscopy, implementation of revised clinical practice (routine blood test for all febrile adults and restricting treatment to only positive tests) reduced the financial costs for antimalarial drugs, antibiotics, 
microscopy, and errors from over-diagnosing malaria by almost $60 \% .{ }^{8} \mathrm{~A}$ study in Uganda among febrile children with a negative smear test for malaria, who were followed up without being given an antimalarial drug, found that less than $1 \%$ developed uncomplicated malaria and identified no unfavourable outcomes. ${ }^{9}$ Withholding treatment for malaria in people with a negative test was safe and saved treatments for children in more urgent need. ${ }^{9}$

So what can we learn from the study by Reyburn and colleagues? Ideally the findings should "kick start" the process to change the behaviour of health workers. National and international guidelines should be explicit about how to treat patients with negative tests. The choice between rapid diagnostic tests or microscopy will depend on local circumstances, including available skills, the use of microscopy for the diagnosis of other diseases, and whether patients seek treatment from formal health facilities or from community health workers. Innovative approaches beyond basic training will be needed, including regular supervision and team building between laboratory and clinical staff, regular consensus reviews, surveillance and trend analysis for laboratory confirmed malaria and other common febrile illnesses, and interventions to increase public knowledge about the right way to diagnose malaria.

References are on bmj.com

\section{Oral chemotherapy}

\section{Standardised dosing can improve the safety of prescribing}

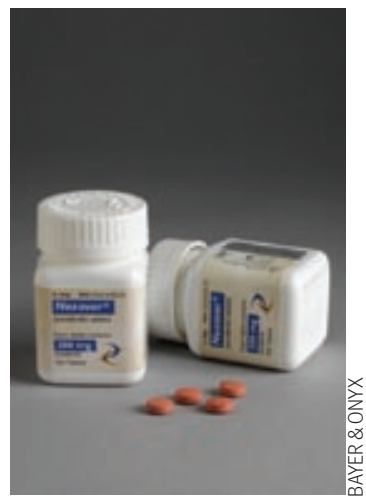

RESEARCH p 407

Sandeep D Parsad clinical haematology and oncology pharmacis

sandeep.parsad@uchospitals.edu Mark J Ratain Leon O Jacobson professor of medicine

Department of Medicine, Section of Haematology/Oncology, University of Chicago, Chicago, Illinois 60637, USA

Competing interests: None declared. Provenance and peer review: Commissioned; not externally peer reviewed.

BMJ 2007;334:376 doi: 10.1136/bmi.39128.449317.BE
The use of oral anticancer agents for the treatment of common malignancies has increased over the past few years. Of about 300 new anticancer agents in development, $20-25 \%$ are oral products. ${ }^{1}$ Many novel "target agents" such as inhibitors of the epidermal growth factor receptor (for example, erlotinib) and the vascular endothelial growth factor receptors (for example, sunitinib and sorafenib) are given orally. Patients prefer oral agents because they are more convenient, allow greater autonomy, and avoid venepuncture and the associated risks of indwelling venous catheters. ${ }^{2}$

Despite the advantages of oral anticancer agents, they do pose challenges such as poor compliance, a small but definite risk of unintentional overdose, and a greater risk of drug-drug and drug-food interactions. ${ }^{3}$ In this week's BMJ, Weingart and colleagues evaluate the safety practices of 62 National Cancer Institute designated centres in the United States with regard to prescribing oral chemotherapy. ${ }^{4}$ Such institutions would be expected to have high safety standards, but the survey found that few of the safeguards suggested for parenteral anticancer drugs were used for oral agents given in an ambulatory setting. More than half of the responding centres (23 of 42) had no required element for oral chemotherapy prescriptions; these included the diagnosis, protocol number, cycle number on the prescription, or a double check of the prescription by a second clinician. ${ }^{4}$

Since 1996, most US hospitals have devised programmes for the safe delivery of oral and parenteral chemotherapy. ${ }^{5}$ Recommendations emphasise the need for a comprehensive, interdisciplinary approach to reduce the number of errors whereby the accuracy of chemotherapy prescriptions must be verified by a system of double checking. ${ }^{67}$ Weingart and colleagues' survey suggests that these safety recommendations often assume chemotherapy agents will be administered in a monitored, clinical environment, and not in an ambulatory setting. Oral anticancer agents given outside a healthcare centre require the same safeguards as parenteral agents, unless the dosing regimen of oral agents can be simplified.

One source of prescribing error stems from the unique practice in oncology of calculating doses on the basis of the patient's body surface area (calculated from height and weight). This practice has been repeatedly questioned in recent years, and the evidence to support its use in clinical practice is limited. ${ }^{8-10}$

The rationale for using body surface area to calculate drug doses is even less clear for oral agents than for parenteral ones, as pharmacokinetic variability between patients is greater for oral agents and less likely to be associated with body size. ${ }^{1112}$ Thus, calculating the dose of oral anticancer agents on this basis is unlikely to improve safety and may even increase the risk of underdosage or overdosage. Some drug companies have responded to these risks by creating standard starting doses for all patients. However, some widely used oral agents (such as capecitabine) still require dosing on the basis of body surface area. $^{13}$

For doses based on body surface area, safety standards should be the same as for parenteral therapy-the prescription should include the patient's height, weight, and body surface area; dose per body surface area; final calculated dose; and total number of doses per treatment course. Dosing calculations should be verified by a multidisciplinary system of double checking. ${ }^{6-8}$

Prescriptions for agents with standardised dosing may not need these elaborate safety measures as the risks of the prescriber making a dosing error or the patient making an error when taking the drug are much lower.

The use of oral anticancer agents will continue to increase as more agents come on to the market. We suggest standardising oral anticancer doses in an effort to improve patient safety.

References are on bmi.com 


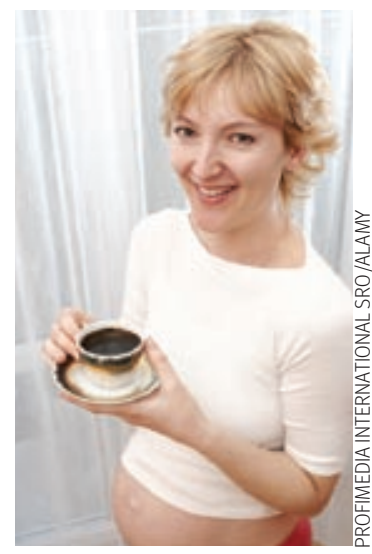

\section{RESEARCH p 409}

Edmund Hey retired paediatrician, Braithwaite, West Yorkshire, BD22 6PX

shey@easynet.co.uk

Competing interests: None declared. Provenance and peer review: commissioned; not externally peer reviewed.

BMJ 2007;334:377 doi: 10.1136/bmj.39122.395058.80

\section{Coffee and pregnancy}

\section{A moderate reduction in caffeine intake in the second half of pregnancy has no effect on birth weight or length of gestation}

Some like their coffee black, and some like it white, but whether it is wise to drink coffee in pregnancy is not a black and white issue. Many observational studies have suggested that it is unwise to drink coffee (or indeed any drink containing caffeine) during pregnancy. Some papers report that consumption of more than modest amounts of caffeine during pregnancy may increase the likelihood of infertility, birth defects, miscarriage, stillbirth, premature birth, fetal growth restriction, and cot death. Each such paper has spawned a flurry of further papers reporting a failure to find any such association. One recent review article cited more than 200 papers. ${ }^{1}$ The problem is that women who drink more coffee than most nearly always differ from other pregnant women in other ways too. They are more likely to smoke, for one thing, which makes it difficult to decide what is causing what. ${ }^{2}$

In this week's $B M J$, we finally have an interventional study by Bech and colleagues ${ }^{3}$ showing that babies born to mothers who drink moderate amounts of coffee do not weigh less than those whose mothers' drink decaffeinated coffee in the second half of pregnancy (as 12 observational studies had previously suggested ${ }^{2}$.

Caffeine crosses the placenta easily, and the speed with which it is then metabolised declines during pregnancy. Exposure to artificial boluses of caffeine can certainly damage the fetal rat, but only when the amount is 10 times higher than any human would ever ingest, even if they drank nothing but the most potent caffeinated beverage in a dose high enough to render them ill. ${ }^{1} \mathrm{~A}$ widely quoted paper in the Lancet in 1988 suggested that "women who consumed more than the equivalent of one cup of coffee per day were half as likely to become pregnant per cycle as women who drank less." ${ }^{4}$ However, the nine studies that have since looked into this unexpected finding found little evidence to support this conclusion once other influ-

Common drinks and foods and their typical caffeine content

\begin{tabular}{ll} 
Drink or food & Caffeine content \\
\hline Cola and other “energy" drinks & $12-60 \mathrm{mg} / 300 \mathrm{ml}$ can \\
\hline Bottled iced tea & $15-25 \mathrm{mg} / 300 \mathrm{ml}$ bottle \\
\hline Brewed tea (non-herbal)* & $20-50 \mathrm{mg} / \mathrm{cupt}$ \\
\hline Mate (South American tea) & $30-60 \mathrm{mg} / \mathrm{cup}$ \\
\hline Decaffeinated coffee & $4-8 \mathrm{mg} / \mathrm{cup}$ \\
\hline Instant coffee & $40-140 \mathrm{mg} / \mathrm{cup}$ \\
\hline Brewed coffee & $60-200 \mathrm{mg} / \mathrm{cup}$ \\
\hline Chocolate & $5-35 \mathrm{mg} / 50 \mathrm{~g}$ bar \\
\hline
\end{tabular}

*Twenty per cent more than this for tea brewed for more than 3 minutes. tTaken to be a $300 \mathrm{ml}(\sim 10 \mathrm{fl} \mathrm{oz})$ cup. ences such as maternal age, smoking, and parity were taken into account. ${ }^{2}$

Early miscarriage is certainly more common in women who drink substantial amounts of coffee in early pregnancy. ${ }^{5}$ However, we do not know whether continuing high consumption puts the fetus at risk, or whether sustained consumption is simply a marker for a pregnancy that is already doomed, because an increased aversion to coffee is, along with nausea and vomiting, a consistent early feature of a healthy pregnancy. ${ }^{6}$

The report of a dose dependent relation between intake of caffeine before pregnancy and the risk of miscarriage suggests that a very high intake of caffeine prenatally may be unwise, but the adjusted odds ratio when the 186 women taking less than $75 \mathrm{mg}$ a day were compared with the 230 taking more than $900 \mathrm{mg}$ a day was only just significant $(1.72 ; 95 \%$ confidence interval 1.00 and 2.96). ${ }^{7}$

Caffeine consumption does not make preterm birth more likely, ${ }^{28}$ and the only report of a link between consumption in late pregnancy and cot death could not be replicated. ${ }^{2}$ However, a paper in the $B M J$ in 2003 did report an excess of fetal death in the second half of pregnancy in Danish women who said at booking that they drank eight or more cups of coffee a day. So too did a subsequent study of women who said they drank four or more cups a day that used data from a national data set. ${ }^{9}$ A recent study in Uruguay, which did not fully adjust for smoking status, had similar findings. ${ }^{10}$

Estimating fetal exposure is more difficult than is thought because cup size and the way the drink is prepared vary more than is realised. The caffeine content of different brands of tea and coffee also varies, and these drinks are not the only important dietary sources of caffeine (table). ${ }^{11} 12$ In addition, the speed with which caffeine is cleared by the liver varies greatly. Clearance occurs more rapidly in smokers and is affected by a range of genetic polymorphisms affecting cytochrome P450 1A2. ${ }^{11}$

The US Food and Drug Administration has been advising women to avoid or limit their intake of caffeine in pregnancy since 1980, and the UK Food Standards Agency issued slightly more nuanced advice in 1984, which it updated in October 2001. The randomised controlled trial by Bech and colleagues ${ }^{3}$ should lead to revision of this advice, at least with regard to birth weight. But we now need a similar, larger trial to show whether observational studies are right in suggesting that a high intake of caffeine increases the risk of stillbirth.

References are on bmi.com 


\section{Managing suspected research misconduct}

\section{Authors, editors, and systematic reviewers should protect the public from unsound research data}

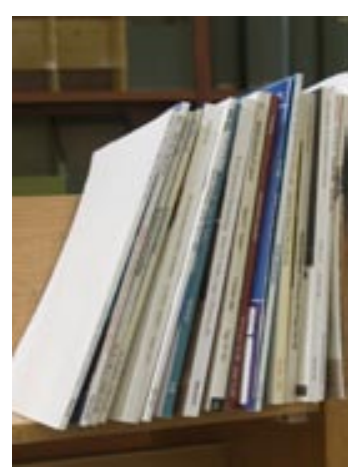

\section{FEATURE $p 392$}

Charles Young editor, BMJ Clinical Evidence charles.young@bmigroup. com Fiona Godlee editor, BM] BMJ, London WC1H 9JR

Competing interests: $\mathrm{CY}$ is editor of BMJ Clinical Evidence which produces systematic reviews. FG is editor of the $B M$ J, former president of WAME, and former chair of COPE. Provenance and peer review: Commissioned; not externally peer reviewed

BMJ 2007;334:378-9 doi: 10.1136/bmj.39129.611516.80
In this week's BMJ, Ian Roberts, Richard Smith, and Stephen Evans describe the worrying story of Dr Julio Cruz. ${ }^{1}$ Cruz, a previously highly regarded medical researcher and clinician, committed suicide two years ago. Three of his publications about the use of high dose mannitol in head injury have recently been called into question. Furthermore, his coauthors and the editors of the journals in which the three papers were first published have failed to respond adequately to concerns raised about the integrity of the data in these papers.

These events have several important consequences. Many doctors base key treatment decisions on the results of published randomised trials. If some or all of Cruz's data on high dose mannitol are false, then doctors will be providing their critically ill patients with uncertain and possibly harmful treatment. In doing so, those doctors will also deny their patients other treatments that are based on reliable evidence.

The failure to retract unsound data also has long term consequences as the data become integrated into reviews, meta-analyses, and guidelines. These syntheses of primary research affect the practice of clinicians worldwide, and in turn affect even larger numbers of patients. In addition, unless and until the veracity of Cruz's data is formally proved or disproved, there is a risk that further research will not be conducted in this area. This again denies practising clinicians the possibility of access to the sound data they need.

Concerns about the authenticity of biomedical research data are increasingly being publicised. Recent high profile examples include publications by Jon Sudbo ${ }^{2}$ and Hwang Woo-suk, ${ }^{3}$ both of whom have had their results discredited or retracted. Importantly, however undesirable the publication of unsound data is, the consequences of such publication are made far worse by the subsequent failure of the people involved to react appropriately to valid concerns and correct the scientific record where necessary.

Part of the difficulty in dealing with Cruz's data relates to his tragic death before the start of any formal investigation. As with at least one other prominent case of alleged misconduct, ${ }^{4}$ he belonged to no institution that could be charged with undertaking the necessary investigation. In the wake of Cruz's death, any reasonable person would assume that the responsibility for the disputed publications rested with the other investigators whose names appeared alongside his on the original papers. These individuals are coauthors of the published papers as defined by the International Committee of Medical Journal Editors, ${ }^{5}$ or at least contributors, ${ }^{6}$ and have themselves denied they were "gift authors." Where they are unable to verify the findings with which their names are associated, they have a clear obligation, in our view, to take all necessary steps to correct the record. To date they have failed in this duty.

Several groups already provide guidelines on how editors should react if research misconduct, including publication of false data, is suspected. These groups include medical journals, ${ }^{78}$ the Committee on Publication Ethics, ${ }^{9}$ and the World Association of Medical Editors. ${ }^{10}$ In broad terms, editors are advised to discuss the situation with the authors involved. If this discussion does not produce a satisfactory result the situation should be referred to an appropriate higher authority-perhaps the authors' academic institution or funding body. If, after an appropriate investigation, it is shown that false data have been published, the data should be retracted. Of note, the Committee on Publication Ethics makes particular mention that editors should react promptly to alert readers in situations where inaccuracies or misleading statements may have been published.

It is clear from communications between Roberts and John Jane, editor of the Journal of Neurosurgery (which published one of the disputed Cruz papers), that Jane doubted the veracity of the Cruz data. However, an accompanying editorial in that journal by Marshall only alluded to general problems with single centre research studies. Jane did not inform readers that he did not trust the Cruz data and indeed suspected that it was fabricated. ${ }^{11}$

Despite making considerable efforts, both Roberts and the BMJ Clinical Evidence editorial team have had great difficulty in contacting Michael Apuzzo, editor of Neurosurgery, in connection with the Cruz data. Neurosurgery published the two other disputed Cruz papers, and Apuzzo seems reluctant to address the serious criticisms of his journal's content. In failing to alert readers promptly to concerns about the Cruz data published in their journals, Jane and Apuzzo have created confusion. The position ought to be made clear in the interests of patients around the world.

It has been left to Roberts, in his capacity as coordinating editor of the Cochrane injuries group, and his colleagues to investigate and attempt to resolve the tangle of claims and counter claims that surround Cruz's data, and then to bring them out into the open. Their investigation of the three disputed papers should act as a model to which future systematic reviewers could usefully aspire. Systematic reviews, with or without meta-analyses, are appropriately replacing single clinical trials as agents that change and shape clinical practice. Consequently, researchers, such as Roberts, and organisations, such as the Cochrane Collaboration, that produce reviews have a growing responsibility to ensure that the data they summarise are valid. If done consistently, this assessment would become another 
important check on dubious information remaining as part of the evidential record on which global health care is based. However, efforts such as Roberts' do not affect the primary responsibility of journal editors to investigate thoroughly for themselves when serious suspicions of research misconduct are brought to their attention, and when doubts cannot be dispelled to make appropriate amendment to the scientific record. ${ }^{9}$

Bodies do exist in some countries that either directly undertake investigations into research misconduct (for example, the Danish Committees on Scientific Dishonesty (http://fist.dk/site/english/councils-commissions-committees/the-danish-committees-on-scientific-dishonesty)) or indirectly support investigation by institutions (for example, the US Office of Research Integrity (http://ori.dhhs.gov) and the recently launched UK Panel for Research Integrity (http:// www.ukrio.org)). However, international awareness about research misconduct, though growing, is still low. Roberts and colleagues propose the creation of an international group with responsibility for dealing with research misconduct.

In the case of Cruz's data, more immediate steps must be taken to protect patients with head injuries. The editors and coauthors involved in the disputed Cruz papers must act quickly either to confirm the veracity of what they have published or to withdraw it. In the interim, clinicians treating patients with head injuries should approach these data with caution. Finally, in the absence of any patently sound evidence for its effectiveness, the research community should be encouraged to subject high dose mannitol to further research.

References are on bmj.com
P M Rothwell director, stroke prevention research unit, Radcliffe Infirmary, University of Oxford, Oxford OX2 6HE

peter.rothwell@clneuro.ox.ac.uk

Competing interests: None declared.

BMJ 2007;334:379-80 doi: 10.1136/bmj.38964.489051.80

\section{Atherothrombosis and ischaemic stroke}

\section{Unstable plaque is the main mechanism of stroke in patients with carotid stenosis}

Thrombosis due to "unstable" atherosclerotic plaque is the main mechanism underlying acute coronary syndromes, and vascular research has focused mostly on this model. Plaque also causes a substantial proportion of ischaemic stroke, although multiple mechanisms are involved and "stable" plaque is sometimes responsible. For example, in the basilar and proximal middle cerebral arteries, stroke can result from occlusion of a small branch vessel by slow growth of otherwise "stable" plaque in the parent vessel. Slowly growing but stable plaque can also cause cerebral ischaemia due to stenosis and hypoperfusion without thromboembolism. Recent evidence, however, suggests that the predominant mechanism of stroke, at least in patients with carotid stenosis, is similar to the coronary model and involves mainly unstable plaque. ${ }^{1-3}$ This observation has implications for the way we manage and prevent strokes.

Carotid plaques are typically slow growing or quiescent for long periods but may suddenly develop ruptures, fissures, or endothelial erosions, triggering platelet aggregation and formation of thrombus, which leads to local occlusion or embolisation to more distal vessels. Recent studies have correlated histology of the plaque with time since last symptoms in patients with symptomatic stenosis undergoing endarterectomy. ${ }^{2}{ }^{3}$ Spagnoli and colleagues studied 187 symptomatic plaques and reported that the frequency of thrombotically active plaque was greater after stroke than after transient ischaemic attack, and that it fell with time from first ischaemic symptoms to surgery. ${ }^{2}$ A similar study of 565 symptomatic carotid plaques found a high frequency of features that mark unstable plaque (for example, rupture of cap in $56.7 \%$, a large lipid core in $59.6 \%$, marked inflammatory infiltrate in $66.9 \%$ ) and found that many of these features, particularly inflammation, were most frequent in patients with recent cerebral ischaemic events, especially after stroke. ${ }^{3}$ Interestingly, cap thickness in ruptured carotid plaques is much greater than that reported in ruptured coronary plaques, ${ }^{3}$ which has implications for identifying at risk plaques by imaging. However, rupture is still associated with a relatively thin cap and with pronounced macrophage infiltration. ${ }^{3}$

The finding that coronary-type "unstable" plaque is responsible for a high proportion of transient ischaemic attacks and strokes in patients with carotid stenosis has important implications for prevention. Firstly, it highlights the need for urgency in investigation and treatment. The risk of major stroke distal to symptomatic carotid stenosis is up to 30\% during the first month after the presenting event, ${ }^{4}$ but this risk falls rapidly with time, as does benefit from endarterectomy. ${ }^{5}$ In relevant trials, for patients with $50 \%$ or higher stenosis, the number needed to undergo surgery (number needed to treat) to prevent one ipsilateral stroke in five years was five for patients randomised within two weeks after their last ischaemic event versus 125 for patients randomised after 12 weeks. ${ }^{5}$ Unfortunately, the current average delay before endarterectomy in the United Kingdom is about 12 weeks, ${ }^{4}$ and many patients have a major stroke before investigation or surgery. The rapid fall in risk of stroke with time since presenting event could be due to the development of collateral circulation or the loss of a small subgroup of patients who are particularly susceptible to stroke for some other reason, but this fall is most likely to be due to healing of unstable plaque.

Secondly, the role of unstable plaque in the aetiology of ischaemic stroke indicates that sheer induced platelet aggregation could be involved in thrombus formation and that antiplatelet agents may 


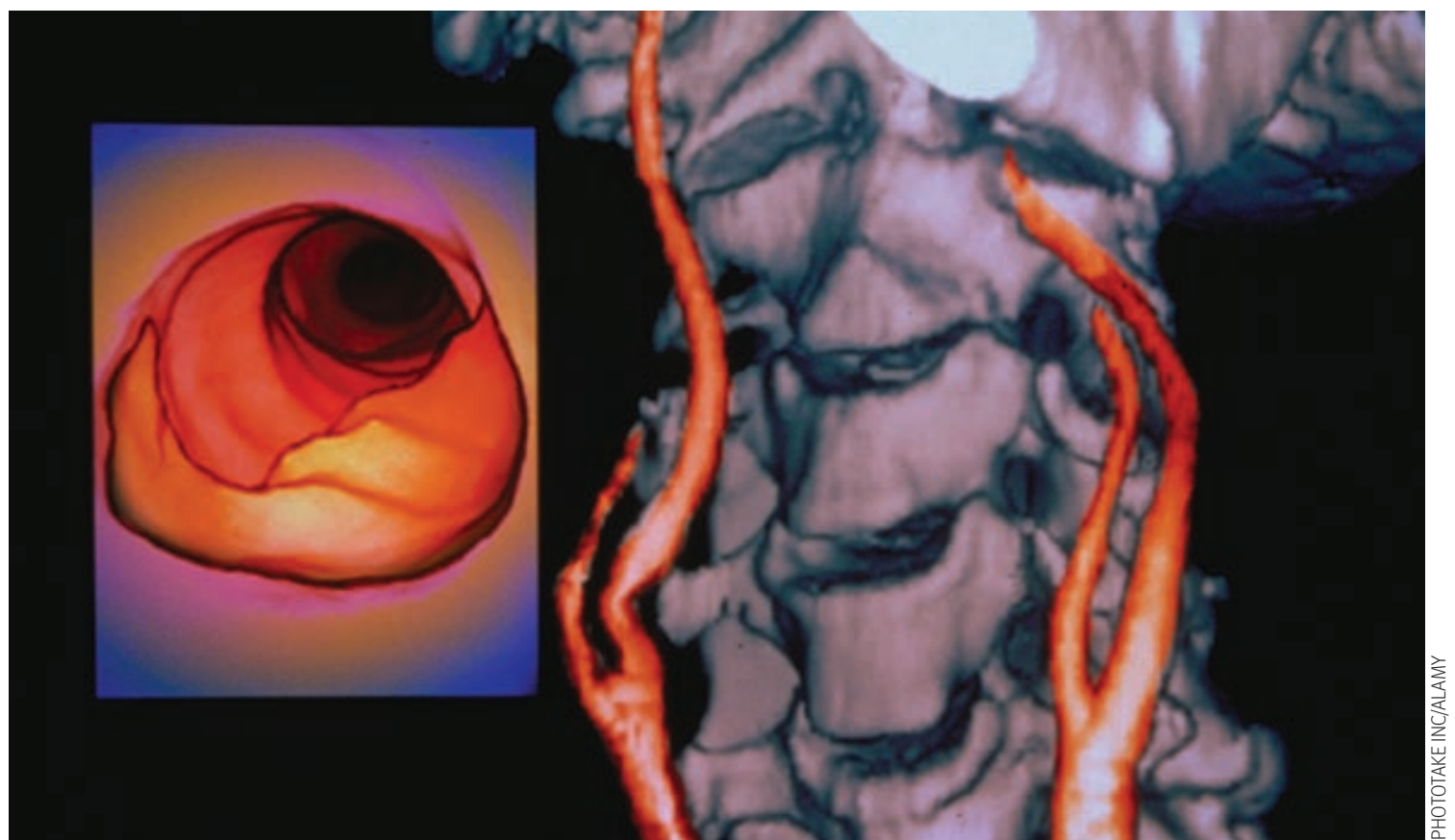

have potential in the prevention of ischaemic stroke. This is consistent with recent data on the potential benefit of a short course of combinations of antiplatelet agents in patients with acutely symptomatic carotid stenosis, ${ }^{6}$ and the lack of efficacy of warfarin in patients with intracranial stenosis. ${ }^{7}$

There are also important implications for research. Firstly, unstable carotid plaque can be imaged in vivo, so imaging might have a role in risk stratification. Ulceration of the surface of the plaque on conventional arterial angiography, which is strongly associated with unstable plaque on histology, ${ }^{1}$ is a strong independent predictor of stroke, ${ }^{5}$ and has been included in risk models for patients with symptomatic carotid stenosis. ${ }^{8}$ Carotid ultrasound can identify lipid-rich echolucent plaques, and magnetic resonance imaging can detect both lipid core and intraplaque haemorrhage, although more research is needed to determine whether these assessments predict stroke. Novel imaging techniques using magnetic resonance imaging, positron emission tomography, and molecular radiolabelling also allow quantification of macrophage infiltration, neovascularisation, metabolic activity, and even protease activity and apoptosis. ${ }^{9}$

Secondly, carotid plaques also provide an indirect window on the coronary circulation. Non-invasive measurements of carotid stenosis can predict severe coronary artery disease in patients with suspected ischaemic heart disease and future acute coronary events in patients with coronary artery disease. Importantly, ruptured carotid plaques are more likely than smooth plaques to be associated with future coronary events, ${ }^{10}$ which suggests that plaque instability is a systemic phenomenon and that non-invasive assessment of carotid plaque instability might also be a useful index of coronary risk.

Thirdly, the importance of instability of stenosing carotid plaques as a cause of transient ischaemic attack and stroke raises the possibility that, as in the coronary circulation, many acute carotid ischaemic events might be caused by instability in non-stenosing plaque. Although the average risk of stroke in patients without appreciable carotid stenosis is insufficient to merit endarterectomy, recent developments in imaging techniques allow the subgroup of patients with unstable plaques to be better identified ${ }^{9}$ and will necessitate further research to determine optimal treatment.

Finally, most of what we know about atherosclerotic plaque and stroke relates to disease at the carotid bifurcation-the only arterial segment routinely imaged after a stroke or transient ischaemic attack in most centres. However, many other points of branching, tortuosity, or confluence of the arterial supply to the brain are also prone to disease. Common sites of extracranial atheroma include the aortic arch, where large plaques are an important risk factor for ischaemic stroke ${ }^{11}$; the proximal subclavian and common carotid arteries; and the origins of the vertebral arteries. ${ }^{12}$ The intracranial arteries are prone to atherosclerosis at the carotid siphon, the proximal middle cerebral artery, the intracranial vertebral arteries, and the basilar artery origin. ${ }^{13} 14$ Although intracranial disease does appear to be associated with a high risk of recurrent stroke on medical treatment, ${ }^{7}$ the proportion of all strokes caused by plaque at sites other than the carotid bifurcation is unknown and will differ between populations. Carotid and other extracranial atherosclerosis is most common in white men, whereas intracranial disease is most common in black, Hispanic, and oriental populations, in patients with type 1 diabetes, and in younger women. ${ }^{13}{ }^{14}$ More research is required into imaging and treatment of disease at sites other than the carotid bifurcation, particularly in patients with ischaemic events in the posterior circulation, in whom the early risk of recurrent stroke is probably as high as in patients with carotid stenosis. ${ }^{15}$

References are on bmj.com 